\title{
ISOSPECTRAL HYPERSURFACES IN EUCLIDEAN SPHERES
}

\author{
José N. B. BARBOSA
}

\begin{abstract}
The aim of this work is to present a classification of some compact hypersurfaces $M^{n}$ of a unit sphere $S^{n+1}$ provided the spectra of the Laplacian of $p$-forms of $M^{n}$, which we denote by $\operatorname{Spec}^{p}(M)$, is equal to the $\operatorname{spectra} \operatorname{Spec}^{p}\left(M_{0}\right)$, of a given hypersurface $M_{0}^{n}$.
\end{abstract}

\section{Introduction}

Let $M$ be a compact Riemannian manifold without boundary of dimension $n$. We will denote the spectrum of the Laplacian of $p$-forms in $M$ by

$$
\operatorname{Spec}^{p}(M):=\left\{0 \leq \lambda_{0}^{p} \leq \lambda_{1}^{p} \leq \cdots \uparrow+\infty\right\}, \quad p=0,1, \ldots, n .
$$

One hard problem in Riemannian Geometry is to decide whether two isospectral Riemannian manifolds are isometric. The existence of flat tori which are isospectral but are not isometric (see [3]) is a counterexample to the validity in general of a positive answer to this question. The principal ingredient used to deal with this problem is the asymptotic expansion formula of the heat kernel due to Minakshisundaram-Pleijel (see [3] or [8]) which asserts

$$
\sum_{i=1}^{\infty} e^{-\left(\lambda_{i}^{p}\right) t} \sim(4 \pi t)^{-n / 2}\left(a_{0, n}^{p}+a_{1, n}^{p} t+a_{2, n}^{p} t^{2}+\cdots\right), \quad t \rightarrow 0^{+},
$$

where $a_{i, n}^{p}$ are geometric constants depending on $M$.

However, if we consider an isometric immersion of $M$ into the Euclidean sphere $S^{n+1}$ with some geometric properties, this problem comes less dificult. For instance, Q. Ding [7] proved that if $M$ is a closed, orientable minimal hypersurface of $S^{4}$ and $\operatorname{Spec}^{p}(M)=\operatorname{Spec}^{p}\left(M_{0}\right)$, for a given $p \in\{0,1,2,3\}$, where $M_{0}$ is the totally geodesic sphere, or the Clifford torus $S^{1}(\sqrt{1 / 3}) \times S^{2}(\sqrt{2 / 3})$, or the Cartan minimal hypersurface, then $M$ is isometric to $M_{0}$. On the other hand, J. Wang [10] had shown that if $M$ is a closed, orientable hypersurface in $S^{4}$ with constant mean curvature $H, M_{H}$ is an isoparametric hypersurface in $S^{4}$ with

Mathematics Subject Classifications (1991): 53 A 10, 53 C 42.

Received August 8, 2003. 
the same mean curvature $H$ and $\operatorname{Spec}^{p}(M)=\operatorname{Spec}^{p}\left(M_{H}\right), \forall p \in\{0,1\}$, then $M$ is isometric to $M_{H}$.

We will denote by $k_{i}, i=1, \ldots, n$, the principal curvatures of an immersed hypersurface $M \hookrightarrow S^{n+1}$. In that way, the symmetric functions of $k_{i}$ are defined by

$$
\sigma_{m}=\sum_{\substack{i_{1}, \ldots, i_{m}=1 \\ i_{1}<\cdots<i_{m}}}^{n} k_{i_{1}} \cdots k_{i_{m}},
$$

with $m=1, \ldots, n$. The square of the length of the second fundamental form is given by

$$
S=\sum_{i=1}^{n} k_{i}^{2}
$$

Finally, $\mathrm{d} v$ stands for the element of volume of $M$.

Now, we are able to state the main theorem of this work:

THEOREM 1. Let $M, M_{0} \hookrightarrow S^{n+1}, n \geq 3$, be closed hypersurfaces of $S^{n+1}$ with mean curvatures $H$ and $H_{0}$, and scalar curvatures $\rho$ and $\rho_{0}$, respectively. We require that one of the curvatures $H$ and $H_{0}$ is nonnull and $\rho_{0}$ is constant. Suppose in addition that

(i) $\operatorname{Spec}^{p}(M)=\operatorname{Spec}^{p}\left(M_{0}\right), \forall p \in\{0,1\}$, if $n=3$;

(ii) $\operatorname{Spec}^{p}(M)=\operatorname{Spec}^{p}\left(M_{0}\right), \forall p \in\{0,1,2\}$, if $n \geq 4$.

Then $\rho=\rho_{0}$, i.e., $M$ has also the same constant scalar curvature as $M_{0}$. Moreover the following integral equalities hold:

$$
\begin{gathered}
\int_{M} H \sigma_{3} \mathrm{~d} v=\int_{M_{0}} H_{0} \sigma_{3}^{0} \mathrm{~d} v_{0}, \quad \text { if } n \geq 3, \\
\int_{M} \sigma_{4} \mathrm{~d} v=\int_{M_{0}} \sigma_{4}^{0} \mathrm{~d} v_{0}, \quad \text { if } n \geq 4,
\end{gathered}
$$

where $\sigma_{m}^{0}$ and $\mathrm{d} v_{0}$ denote the values of $\sigma_{m}$ and $\mathrm{d} v$ correspondent to $M_{0}$, respectively. In particular, we have

$$
n^{2} H^{2}-S=n^{2} H_{0}^{2}-S_{0},
$$

where $S_{0}$ is the square of the length of the second fundamental form of $M_{0}$.

A consequence of our calculations is the next result about the case $H=H_{0}=0$, whose proof follows closely techniques presented before by Q. Ding in his paper [7].

THeORem 2. Let $M, M_{0} \hookrightarrow S^{n+1}, n \geq 3$, be closed minimal hypersurfaces of $S^{n+1}$ whose scalar curvatures are $\rho$ and $\rho_{0}$, respectively, with $\rho_{0}$ constant. Suppose that 
(i) $\operatorname{Spec}^{p}(M)=\operatorname{Spec}^{p}\left(M_{0}\right)$, for some $p \in\{0,1,2,3\}$, if $n=3$;

(ii) $\operatorname{Spec}^{p}(M)=\operatorname{Spec}^{p}\left(M_{0}\right), \forall p \in\{0,1\}$, if $n \geq 4$.

Then $\rho=\rho_{0}$. Moreover, for $n \geq 4$, we have

$$
\int_{M} \sigma_{4} \mathrm{~d} v=\int_{M_{0}} \sigma_{4}^{0} \mathrm{~d} v_{0}
$$

Given $r \in(0,1)$ and $m \in\{1, \ldots, n-1\}$ we will denote by $M_{n-m, m}^{r}(H)$, the hypersurface of $S^{n+1}$ with constant mean curvature $H$, obtained by considering the standard immersions $S^{n-m}(r) \subset \boldsymbol{R}^{n-m+1}, S^{m}\left(\sqrt{1-r^{2}}\right) \subset \boldsymbol{R}^{m+1}$ of spheres with radius $r$ and $\sqrt{1-r^{2}}$ and dimensions $n-m$ and $m$, respectively, and taking the product immersion

$$
S^{n-m}(r) \times S^{m}\left(\sqrt{1-r^{2}}\right) \hookrightarrow \boldsymbol{R}^{n-m+1} \times \boldsymbol{R}^{m+1} .
$$

Thus we have that $M_{n-m, m}^{r}(H)$ is contained in $S^{n+1}$ and has principal curvatures $k_{i}, i=1, \ldots, n$, and mean curvature, respectively, given by

$$
k_{1}=\cdots=k_{n-m}=\frac{\sqrt{1-r^{2}}}{r}, \quad k_{n-m+1}=\cdots=k_{n}=-\frac{r}{\sqrt{1-r^{2}}},
$$

and

$$
H=\frac{n-m-n r^{2}}{n r \sqrt{1-r^{2}}},
$$

or the negative of these values when we choose the opposite orientation. The hypersurface $M_{n-m, m}^{r}(H)$ is usually known as $H(r)$-torus or generalized Clifford Totus.

Let $\mathscr{F}_{H}$ be the set consisting of isoparametric hypersurfaces in $S^{4}$ with constant mean curvature $H$. E. Cartan proved in [5] that if $M \in \mathscr{F}_{H}$ then $M$ is totally umbilical, or a $H(r)$-torus $M_{3-k, k}^{r}(H)$, or a Cartan hypersurface (that is, the isoparametric hypersurface obtained from the Cartan minimal hypersurface). Using Theorem 1 we will show that the assumption $H=H_{0}$ is not necessary in the theorem proved by J. Wang, above mentioned. More precisely, we will prove the following result:

THEOREM 3. Let $M \hookrightarrow S^{4}$ be a closed and orientable hypersurface with constant mean curvature in $S^{4}$ and $M_{0} \in \mathscr{F}_{H_{0}}$. If $\operatorname{Spec}^{p}(M)=\operatorname{Spec}^{p}\left(M_{0}\right)$, for $p \in\{0,1\}$, then $H=H_{0}$ and $M$ is isometric to $M_{0}$. result:

For dimension $n \geq 4$, we will derive also from Theorem 1 the following

THEOREM 4. Let $M \hookrightarrow S^{n+1}, n \geq 4$, be a closed and orientable hypersurface in $S^{n+1}$ with the same constant mean curvature $H_{0}$ of an isoparametric hypersurface $M_{0}$ in $S^{n+1}$. If $\operatorname{Spec}^{p}(M)=\operatorname{Spec}^{p}\left(M_{0}\right), \forall p \in\{0,1,2\}$, then $M$ is also isoparametric. Moreover, 
(i) if $M_{0}$ is either totally umbilical or the $H_{0}(r)$-torus $M_{n-1,1}^{r}\left(H_{0}\right)$, with $r^{2} \leq(n-1) / n$, then $M=M_{0}$.

(ii) When $n=4$ the principal curvatures of $M$ and $M_{0}$ coincide.

Finally, we will prove the following theorem:

THEOREM 5. Let $M \hookrightarrow S^{n+1}$ a closed hypersurface of $S^{n+1}$ with nonnegative sectional curvature and $M_{0} \hookrightarrow S^{n+1}$ a totally umbilical hypersurface or a $H_{0}\left(r_{0}\right)$ torus $M_{n-1,1}^{r_{0}}\left(H_{0}\right)$, with $r_{0} \leq(n-2) / n$. Suppose that

(i) $\operatorname{Spec}^{p}(M)=\operatorname{Spec}^{p}\left(M_{0}\right), \forall p \in\{0,1\}$, if $n=3$;

(ii) $\operatorname{Spec}^{p}(M)=\operatorname{Spec}^{p}\left(M_{0}\right), \forall p \in\{0,1,2\}$, if $n \geq 4$.

Then $M$ is isometric to $M_{0}$.

\section{Preliminaries}

Let $M \subset S^{n+1}$ be a closed hypersurface with mean curvature $H$. Choose a local orthonormal frame field $\left\{e_{1}, \ldots, e_{n}\right\}$ and let $\left\{\omega_{1}, \ldots, \omega_{n}\right\}$ be the corresponding dual frame. We consider the second fundamental form

$$
h=\sum_{i, j=1}^{n} h_{i j} \omega_{i} \omega_{j} .
$$

Let $R$ and $\tilde{R}$ be respectively the curvature and Ricci curvature tensors of $M$ and denote by $R_{i j k l}$ and $\tilde{R}_{i j}, i, j, k, l=1, \ldots, n$, their respective components with respect to the above frame. If we choose $\left\{e_{1}, \ldots, e_{n}\right\}$ such that $h_{i j}=k_{i} \delta_{i j}$, then

$$
\begin{gathered}
H=\frac{1}{n} \sum_{i=1}^{n} k_{i}, \\
R_{i j k l}=\left(1+k_{i} k_{j}\right)\left(\delta_{i k} \delta_{j l}-\delta_{i l} \delta_{j k}\right), \\
\tilde{R}_{i j}=\left[(n-1)+n H k_{i}-k_{i} k_{j}\right] \delta_{i j} .
\end{gathered}
$$

Let $\rho$ and $S$ be, respectively, the scalar curvature of $M$ and the square of the length of the second fundamental form $h$. The Gauss formula yields

$$
\rho=n(n-1)+n^{2} H^{2}-S
$$

and taking into account (3) and (4) we have

$$
\begin{aligned}
|R|^{2}= & 2 S^{2}-2 f_{4}+4 n^{2} H^{2}-4 S+2 n(n-1), \\
|\tilde{R}|^{2}= & n^{2} H^{2} S+f_{4}+n(n-1)^{2}-2 n H f_{3} \\
& +2 n^{2}(n-1) H^{2}-2(n-1) S
\end{aligned}
$$


where

$$
f_{m}=\sum_{i=1}^{n} k_{i}^{m}
$$

The expressions of $f_{m}$ can be calculated using the formulas (see e.g. [9], p. 101)

$$
\begin{gathered}
f_{m}-f_{m-1} \sigma_{1}+f_{m-2} \sigma_{2}-\cdots+(-1)^{m-1} f_{1} \sigma_{m-1}+(-1)^{m} m \sigma_{m}=0, \quad \text { for } m \leq n, \\
f_{m}-f_{m-1} \sigma_{1}+\cdots+(-1)^{n} f_{m-n} \sigma_{n}=0, \quad \text { for } m>n .
\end{gathered}
$$

When $n=3$ we get

$$
\begin{aligned}
& f_{3}=\frac{9}{2} H S-\frac{27}{2} H^{3}+3 \sigma_{3}, \\
& f_{4}=\frac{1}{2} S^{2}+9 H^{2} S-\frac{81}{2} H^{4}+12 H \sigma_{3},
\end{aligned}
$$

and for $n \geq 4$,

$$
\begin{aligned}
& f_{3}=\frac{3 n}{2} H S-\frac{n^{3}}{2} H^{3}+3 \sigma_{3}, \\
& f_{4}=\frac{1}{2} S^{2}+n^{2} H^{2} S-\frac{n^{4}}{2} H^{4}+4 n H \sigma_{3}-4 \sigma_{4} .
\end{aligned}
$$

If $H$ is constant, the Simons formula for $M$ is given by

$$
\frac{1}{2} \Delta S=|\nabla h|^{2}+S(n-S)-n^{2} H^{2}+n H f_{3} .
$$

Since $M$ is compact, using Minakshisundaram-Pleijel's asymptotic expansion formula of the heat kernel stated in the introduction we can write

$$
\sum_{i=1}^{\infty} e^{-\left(\lambda_{i}^{p}\right) t} \sim(4 \pi t)^{-n / 2}\left(a_{0, n}^{p}+a_{1, n}^{p} t+a_{2, n}^{p} t^{2}+\cdots\right), \quad t \rightarrow 0^{+},
$$

where

and

$$
\begin{gathered}
a_{0, n}^{p}=\left(\begin{array}{c}
n \\
p
\end{array}\right) \operatorname{vol}(M), \quad a_{1, n}^{p}=\left[\frac{1}{6}\left(\begin{array}{c}
n \\
p
\end{array}\right)-\left(\begin{array}{c}
n-2 \\
p-1
\end{array}\right)\right] \int_{M} \rho \mathrm{d} v \\
a_{2, n}^{p}=\int_{M}\left(E_{n}^{p} \rho^{2}+F_{n}^{p}|\tilde{R}|^{2}+G_{n}^{p}|R|^{2}\right) \mathrm{d} v
\end{gathered}
$$

$$
\begin{aligned}
E_{n}^{p} & =\frac{1}{72}\left(\begin{array}{l}
n \\
p
\end{array}\right)-\frac{1}{6}\left(\begin{array}{l}
n-2 \\
p-1
\end{array}\right)+\frac{1}{2}\left(\begin{array}{c}
n-4 \\
p-2
\end{array}\right) \\
F_{n}^{p} & =-\frac{1}{180}\left(\begin{array}{l}
n \\
p
\end{array}\right)+\frac{1}{2}\left(\begin{array}{l}
n-2 \\
p-1
\end{array}\right)-2\left(\begin{array}{l}
n-4 \\
p-2
\end{array}\right) \\
G_{n}^{p} & =\frac{1}{180}\left(\begin{array}{l}
n \\
p
\end{array}\right)-\frac{1}{12}\left(\begin{array}{l}
n-2 \\
p-1
\end{array}\right)+\frac{1}{2}\left(\begin{array}{l}
n-4 \\
p-2
\end{array}\right),
\end{aligned}
$$


where $\mathrm{d} v$ and $\operatorname{vol}(M)$ represent respectively the volume form and volume of $M$, with respect to the induced Riemannian metric of $S^{n+1}$. We point out that these coefficients were calculated in [8]. Moreover we will decree here that $\left(\begin{array}{l}l \\ q\end{array}\right)=0$ if $l<0$ or $q<0$ or $l<q$.

\section{Proof of Theorems}

We use the same notation for the geometric data of $M$ as in the previous section. We indicate with a subscript " 0 " the corresponding data for $M_{0}$.

Proof of Theorem 1: By hypothesis, the asymptotic expansion formula of $M$ and $M_{0}$ coincide. Thus

$$
\begin{gathered}
\operatorname{vol}(M)=\operatorname{vol}\left(M_{0}\right), \\
\int_{M} \rho \mathrm{d} v=\int_{M_{0}} \rho_{0} \mathrm{~d} v_{0}
\end{gathered}
$$

and

$$
\int_{M}\left(E_{n}^{p} \rho^{2}+F_{n}^{p}|\tilde{R}|^{2}+G_{n}^{p}|R|^{2}\right) \mathrm{d} v=\int_{M_{0}}\left(E_{n}^{p} \rho_{0}^{2}+F_{n}^{p}\left|\tilde{R}_{0}\right|^{2}+G_{n}^{p}\left|R_{0}\right|^{2}\right) \mathrm{d} v_{0} .
$$

Therefore taking in account (5) and (13) we obtain

$$
\int_{M}\left[n(n-1)+n^{2} H^{2}-S\right] \mathrm{d} v=\int_{M_{0}}\left[n(n-1)+n^{2} H_{0}^{2}-S_{0}\right] \mathrm{d} v_{0}
$$

Since

$$
\int_{M} \mathrm{~d} v=\operatorname{vol}(M)=\operatorname{vol}\left(M_{0}\right)=\int_{M_{0}} \mathrm{~d} v_{0}
$$

we conclude that

$$
\int_{M}\left(n^{2} H^{2}-S\right) \mathrm{d} v=\int_{M_{0}}\left(n^{2} H_{0}^{2}-S_{0}\right) \mathrm{d} v_{0} .
$$

We first consider the case $n \geq 4$. Replacing the expressions of $f_{3}$ and $f_{4}$ in (6) and (7) we obtain

$$
\begin{gathered}
|R|^{2}=\left(n^{2} H^{2}-S\right)^{2}+4\left(n^{2} H^{2}-S\right)-8 n H \sigma_{3}+2 n(n-1)+8 \sigma_{4}, \\
|\tilde{R}|^{2}=\frac{1}{2}\left(n^{2} H^{2}-S\right)^{2}+2(n-1)\left(n^{2} H^{2}-S\right)-2 n H \sigma_{3}+n(n-1)^{2}-4 \sigma_{4} .
\end{gathered}
$$

Therefore, for $p \in\{0,1,2\}$, we can write 


$$
\begin{aligned}
& \int_{M}\left(E_{n}^{p} \rho^{2}+F_{n}^{p}|\tilde{R}|^{2}+G_{n}^{p}|R|^{2}\right) \mathrm{d} v \\
& =E_{n}^{p} \int_{M}\left[\left(n^{2} H^{2}-S\right)^{2}+2 n(n-1)\left(n^{2} H^{2}-S\right)+n^{2}(n-1)^{2}\right] \mathrm{d} v \\
& \quad+F_{n}^{p} \int_{M}\left[\frac{1}{2}\left(n^{2} H^{2}-S\right)^{2}+2(n-1)\left(n^{2} H^{2}-S\right)\right. \\
& \left.\quad-2 n H \sigma_{3}+n(n-1)^{2}-4 \sigma_{4}\right] \mathrm{d} v \\
& +G_{n}^{p} \int_{M}\left[\left(n^{2} H^{2}-S\right)^{2}+4\left(n^{2} H^{2}-S\right)-8 n H \sigma_{3}+2 n(n-1)+8 \sigma_{4}\right] \mathrm{d} v .
\end{aligned}
$$

Analogously, we have a similar identity for $M_{0}$. Therefore considering this equations in equality (14) and using (15) we derive the system of equations

$$
\alpha_{n}^{p} \mathbf{X}+\beta_{n}^{p} \mathbf{Y}+\gamma_{n}^{p} \mathbf{Z}=0, \quad p=0,1,2,
$$

where

$$
\alpha_{n}^{p}=\left(E_{n}^{p}+\frac{1}{2} F_{n}^{p}+G_{n}^{p}\right), \quad \beta_{n}^{p}=-2 n\left(F_{n}^{p}+4 G_{n}^{p}\right), \quad \gamma_{n}^{p}=-4\left(F_{n}^{p}-2 G_{n}^{p}\right)
$$

and

$$
\begin{aligned}
\mathbf{X} & :=\int_{M}\left(n^{2} H^{2}-S\right)^{2} \mathrm{~d} v-\int_{M_{0}}\left(n^{2} H_{0}^{2}-S_{0}\right)^{2} \mathrm{~d} v_{0}, \\
\mathbf{Y} & :=\int_{M} H \sigma_{3} \mathrm{~d} v-\int_{M_{0}} H_{0} \sigma_{3}^{0} \mathrm{~d} v_{0}, \\
\mathbf{Z} & :=\int_{M} \sigma_{4} \mathrm{~d} v-\int_{M_{0}} \sigma_{4}^{0} \mathrm{~d} v_{0} .
\end{aligned}
$$

Now a straightforward calculation, using the expressions for $E_{n}^{p}, F_{n}^{p}$ and $G_{n}^{p}$, yields

$$
\operatorname{det}\left(\begin{array}{ccc}
\alpha_{n}^{0} & \beta_{n}^{0} & \gamma_{n}^{0} \\
\alpha_{n}^{1} & \beta_{n}^{1} & \gamma_{n}^{1} \\
\alpha_{n}^{2} & \beta_{n}^{2} & \gamma_{n}^{2}
\end{array}\right) \neq 0 .
$$

We conclude that $\mathbf{X}=\mathbf{Y}=\mathbf{Z}=0$. Therefore,

$$
\begin{gathered}
\int_{M}\left(n^{2} H^{2}-S\right)^{2} \mathrm{~d} v=\int_{M_{0}}\left(n^{2} H_{0}^{2}-S_{0}\right)^{2} \mathrm{~d} v_{0} \\
\int_{M} H \sigma_{3} \mathrm{~d} v=\int_{M_{0}} H_{0} \sigma_{3}^{0} \mathrm{~d} v_{0} \quad \text { and } \quad \int_{M} \sigma_{4} \mathrm{~d} v=\int_{M_{0}} \sigma_{4}^{0} \mathrm{~d} v_{0} .
\end{gathered}
$$


Since $\rho_{0}$ is constant, we have $n^{2} H_{0}^{2}-S_{0}$ constant. Then combining (15), (19) and the Cauchy-Schwarz inequality, we obtain

$$
\begin{aligned}
\left|n^{2} H_{0}^{2}-S_{0}\right| \operatorname{vol}\left(M_{0}\right) & =\left|\int_{M}\left(n^{2} H^{2}-S\right) \mathrm{d} v\right| \\
& \leq\left[\int_{M}\left(n^{2} H^{2}-S\right)^{2} \mathrm{~d} v\right]^{1 / 2}\left[\int_{M} \mathrm{~d} v\right]^{1 / 2} \\
& =\left|n^{2} H_{0}^{2}-S_{0}\right| \operatorname{vol}\left(M_{0}\right) .
\end{aligned}
$$

Thus, $n^{2} H^{2}-S=n^{2} H_{0}^{2}-S_{0}$, which is equivalent to $\rho=\rho_{0}$. This concludes the proof of the theorem for $n \geq 4$.

In the case $n=3$, the calculations are entirely analogous. The similar formula to (18) is given by

$$
\begin{aligned}
\int_{M}\left(E_{3}^{p} \rho^{2}+F_{3}^{p}|\tilde{R}|^{2}+G_{3}^{p}|R|^{2}\right) \mathrm{d} v \\
=E_{3}^{p} \int_{M}\left[\left(9 H^{2}-S\right)^{2}-12\left(9 H^{2}-S\right)+36\right] \mathrm{d} v \\
\quad+F_{3}^{p} \int_{M}\left[\frac{1}{2}\left(9 H^{2}-S\right)^{2}+4\left(9 H^{2}-S\right)-6 H \sigma_{3}+12\right] \mathrm{d} v \\
\quad+G_{3}^{p} \int_{M}\left[\left(9 H^{2}-S\right)^{2}+4\left(9 H^{2}-S\right)-24 H \sigma_{3}+12\right] \mathrm{d} v
\end{aligned}
$$

whereas the corresponding system of equations is

$$
\alpha_{3}^{p} \tilde{\mathbf{X}}+\beta_{3}^{p} \tilde{\mathbf{Y}}=0, \quad p=0,1,
$$

where

$$
\begin{aligned}
\tilde{\mathbf{X}} & :=\int_{M}\left(9 H^{2}-S\right)^{2} \mathrm{~d} v-\int_{M_{0}}\left(9 H_{0}^{2}-S_{0}\right)^{2} \mathrm{~d} v_{0}, \\
\tilde{\mathbf{Y}} & :=\int_{M} H \sigma_{3} \mathrm{~d} v-\int_{M_{0}} H_{0} \sigma_{3}^{0} \mathrm{~d} v_{0} .
\end{aligned}
$$

It is easily checked that

$$
\operatorname{det}\left(\begin{array}{cc}
\alpha_{3}^{0} & \beta_{3}^{0} \\
\alpha_{3}^{1} & \beta_{3}^{0}
\end{array}\right) \neq 0 .
$$

So, proceeding as in the case $n \geq 4$, we complete the proof.

Proof of Theorem 3: It follows from Theorem 1 that $9 H^{2}-S=9 H_{0}^{2}-S_{0}$. Since $H$ is constant, $\mathrm{S}$ is also constant and we can make use the theorems of $\mathrm{S}$. Almeida, F. Brito [2] and S. Chang [5] to conclude that $M$ is isoparametric and 
belongs to $\mathscr{F}_{H}$. In particular $\sigma_{3}$ is also constant. Considering (1) and (12) we conclude

$$
H \sigma_{3}=H_{0} \sigma_{3}^{0} .
$$

We now analyze separatedly three cases.

Case 1) $M_{0}$ is a Cartan hypersurface.

It is known that $S_{0}=6+9 H_{0}^{2}$ and $\sigma_{3}^{0}=-3 H_{0}$ (see e.g. [4]). Thus, using (2) we have $S=6+9 H^{2}$ and, by E. Cartan [4], $M$ is a Cartan hypersurface. By using (21) we obtain $-3 H^{2}=H \sigma_{3}=H_{0} \sigma_{3}^{0}=-3 H_{0}^{2}$, that is, $H= \pm H_{0}$. We now use the same theorem of Cartan [4] to conclude that $M=M_{0}$.

Case 2) $M_{0}$ is totally umbilical.

Since $S_{0}=3 H_{0}^{2}$ and $\sigma_{3}^{0}=H_{0}^{3}$, the expressions (2) and (21) yield

$$
\begin{gathered}
S=9 H^{2}-6 H_{0}^{2}, \\
H \sigma_{3}=H_{0}^{4} .
\end{gathered}
$$

From case 1, it follows that $M$ can not be a Cartan hypersurface, otherwise $M_{0}$ is also a Cartan hypersurface. Since $M \in \mathscr{F}_{H}, M$ is either a $H(r)$-torus $M_{2,1}^{r}(H)$ or totally umbilical. Suppose $M=M_{2,1}^{r}(H)$, for some $r$. Then the principal curvatures of $M$ are

$$
k_{1}=k_{2}=\frac{\sqrt{1-r^{2}}}{r}, \quad k_{3}=-\frac{r}{\sqrt{1-r^{2}}},
$$

or the symmetric of these values for the opposite orientation. We can see now that, independently of the orientation, $H$ and $S$ satisfy

$$
\begin{gathered}
H^{2}=\frac{9 r^{4}-12 r^{2}+4}{9 r^{2}\left(1-r^{2}\right)}, \quad S=\frac{3 r^{4}-4 r^{2}+2}{r^{2}\left(1-r^{2}\right)}, \\
H \sigma_{3}=\frac{3 r^{2}-2}{3 r^{2}} .
\end{gathered}
$$

By using (22) and (24) we conclude that $r^{2}=1 / 3\left(H_{0}^{2}+1\right)<2 / 3$. Hence (25) guarantees $H \sigma_{3}<0$. So, we have a contradiction with (23). Thus, $M$ is totally umbilical and $S=3 H^{2}=9 H^{2}-6 H_{0}^{2}$. Therefore $H= \pm H_{0}$ and similarly $M=M_{0}$.

Case 3) $M_{0}$ is a $H_{0}\left(r_{0}\right)$-torus.

Let us suppose $M_{0}=M_{2,1}^{r_{0}}\left(H_{0}\right)$. From cases (1) and (2) $M$ is neither totally 
umbilical nor Cartan hypersurface. Thus $M$ is an $H(r)$-torus $M_{2,1}^{r}(H)$. It follows from (25) that

$$
H \sigma_{3}=\frac{3 r^{2}-2}{3 r^{2}} \text { and } H_{0} \sigma_{3}^{0}=\frac{3 r_{0}^{2}-2}{3 r_{0}^{2}} .
$$

Since $H$ is constant, (1) and (12) yield $H \sigma_{3}=H_{0} \sigma_{3}^{0}$, from where we conclude that $r=r_{0}$. This finishes the proof of the theorem.

Proof of Theorem 4: First we will consider $H_{0} \neq 0$. Using Theorem 1 for $n \geq 4$ and $H=H_{0}$ we obtain that $S=S_{0}$,

$$
\begin{aligned}
& \int_{M} \sigma_{3} \mathrm{~d} v=\int_{M_{0}} \sigma_{3}^{0} \mathrm{~d} v_{0}, \\
& \int_{M} \sigma_{4} \mathrm{~d} v=\int_{M_{0}} \sigma_{4}^{0} \mathrm{~d} v_{0} .
\end{aligned}
$$

We use now formula (9) to obtain

$$
\begin{aligned}
& f_{3}=\frac{3 n}{2} H_{0} S_{0}-\frac{n^{3}}{2} H_{0}^{3}+3 \sigma_{3}, \\
& f_{3}^{0}=\frac{3 n}{2} H_{0} S_{0}-\frac{n^{3}}{2} H_{0}^{3}+3 \sigma_{3}^{0} .
\end{aligned}
$$

From (26) and the fact that $\operatorname{vol}(M)=\operatorname{vol}\left(M_{0}\right)$ we conclude

$$
\int_{M} f_{3} \mathrm{~d} v=\int_{M_{0}} f_{3}^{0} \mathrm{~d} v_{0}
$$

Since $H=H_{0}, S=S_{0}$ and $\nabla h_{0}=0\left(h_{i j}^{0}\right.$ are constants, $\left.i, j=1, \ldots, n\right)$ the respective Simons formulae (10) for $M$ and $M_{0}$ read as follows

$$
\begin{gathered}
0=\frac{1}{2} \Delta S_{0}=|\nabla h|^{2}+S_{0}\left(n-S_{0}\right)-n^{2} H_{0}^{2}+n H_{0} f_{3}, \\
0=\frac{1}{2} \Delta S_{0}=S_{0}\left(n-S_{0}\right)-n^{2} H_{0}^{2}+n H_{0} f_{3}^{0},
\end{gathered}
$$

from where we conclude that

$$
\int_{M}|\nabla h|^{2}=n H_{0}\left(\int_{M} f_{3} \mathrm{~d} v-\int_{M_{0}} f_{3}^{0} \mathrm{~d} v_{0}\right)=0 .
$$

When $H_{0}=0$ the Theorem 2 carries that $S=S_{0}$ and the Simons formulae for $M$ and $M_{0}$ still imply that $\int_{M}|\nabla h|^{2}=0$. Hence, whatever it is the value of $H_{0}$, we have $\nabla h=0$, that is, $h_{i j k}=0$, for $i, j, k=1, \ldots, n$. Since $M$ is a hypersurface, it follows from formula (2.10) of [6] that

$$
\sum_{k=1}^{n} h_{i j k} \omega_{k}=\mathrm{d} h_{i j}-\sum_{l=1}^{n} h_{i l} \omega_{j l}-\sum_{l=1}^{n} h_{l j} \omega_{i l} .
$$


Since $h_{i j}=k_{i} \delta_{i j}$ and $h_{i j k}=0, i, j, k=1, \ldots, n$, we have

$$
0=\mathrm{d} h_{i j}+\left(k_{i}-k_{j}\right) \omega_{i j}
$$

and setting $i=j$, we conclude $\mathrm{d} k_{i}=\mathrm{d} h_{i i}=0$. Thus, $k_{i}$ is constant, $i=1, \ldots, n$, and $M$ is isoparametric.

On the other hand, the Theorem 1.5 of [1] due to H. Alencar and M. do Carmo gives us that the totally umbilical hypersurfaces of $S^{n+1}$ as well as the $H(r)$-torus $M_{n-1,1}^{r}(H)$ with $r^{2} \leq(n-1) / n$, are characterized by the constant mean curvature and the square of the length of the second fundamental form. Thus, since $H=H_{0}$ and $S=S_{0}$, we can apply the Alencar-do Carmo Theorem to conclude (i).

Let us suppose now that $n=4$ to prove (ii). Since $M$ is isoparametric, $\sigma_{3}$ and $\sigma_{4}$ are both constants. Joining the expressions (26), (27) and the fact that $\operatorname{vol}(M)=\operatorname{vol}\left(M_{0}\right)$ we have $\sigma_{3}=\sigma_{3}^{0}$ and $\sigma_{4}=\sigma_{4}^{0}$. On the other hand,

$$
\sigma_{1}=4 H=4 H_{0}=\sigma_{1}^{0} \quad \text { and } \quad \sigma_{2}=\frac{16 H^{2}-S}{2}=\frac{16 H_{0}^{2}-S_{0}}{2}=\sigma_{2}^{0} .
$$

Therefore the four symmetric functions for $M$ and $M_{0}$ agree and we conclude that $k_{i}=k_{i}^{0}$, for $i=1, \ldots, 4$, which conclude the proof of (ii) of Theorem 4 .

Proof of Theorem 5: It follows from Theorem 1 that $\rho=\rho_{0}$. If $M_{0}$ is totally umbilical, then $\rho_{0}=n(n-1)\left(H_{0}^{2}+1\right)$, whereas for $M_{0}=M_{n-1,1}^{r_{0}}\left(H_{0}\right)$ we have that

$$
\rho_{0}=\frac{(n-1)(n-2)}{r_{0}^{2}} .
$$

It follows in both cases that $\rho_{0} \geq n(n-1)$, i.e., the normalized scalar curvature of $M_{0}$, and hence of $M$, is constant and greater than or equal to 1 . This fact and the assumption that $M$ has nonnegative sectional curvature imply, from Theorem 2 of [11], that $M$ is either totally umbilical or a product of two totally umbilical constantly curved submanifolds. In the last case, $M$ is a $H(r)$ torus. Hence, $H, S$ and $\sigma_{3}$ are constant, as well as $\nabla h=0$. Therefore, Simons formula (10) for $M$ yields

$$
0=S(n-S)-n^{2} H^{2}+n H f_{3} .
$$

The relations (8) and (9) for $M$, allow us to rewrite this formula as

$$
0=S(n-S)-n^{2} H^{2}+\frac{3}{2} n^{2} H^{2} S-\frac{1}{2} n^{4} H^{4}+3 n H \sigma_{3} .
$$

Since $\rho=\rho_{0}$, the Gauss formula implies $S-n^{2} H^{2}=S_{0}-n^{2} H_{0}^{2}=c_{0}$. Then, we have $S=c_{0}+n^{2} H^{2}$ and the equality (29) becomes

$$
0=\left(n-c_{0}\right) c_{0}+n^{2}\left(n-1-\frac{1}{2} c_{0}\right) H^{2}+3 n H \sigma_{3} .
$$


Analogously, the Simons formula for $M_{0}$ give us

$$
0=\left(n-c_{0}\right) c_{0}+n^{2}\left(n-1-\frac{1}{2} c_{0}\right) H_{0}^{2}+3 n H_{0} \sigma_{3}^{0} .
$$

On the other hand, it follows from Theorem 1 that $\int_{M} H \sigma_{3} \mathrm{~d} v=\int_{M_{0}} H_{0} \sigma_{3}^{0} \mathrm{~d} v_{0}$ and with the same argument contained in its proof we conclude $\operatorname{vol}(M)=$ $\operatorname{vol}\left(M_{0}\right)$. Since $H$ and $\sigma_{3}$ are constant, we have that $H \sigma_{3}=H_{0} \sigma_{3}^{0}$. Therefore, putting together the equalities (30) and (31) we obtain

$$
\left(n-1-\frac{1}{2} c_{0}\right)\left(H^{2}-H_{0}^{2}\right)=0 .
$$

We will show that $n-1-(1 / 2) c_{0} \neq 0$. Indeed, otherwise $\rho_{0}=(n-1)(n-2)$, since

$$
\rho_{0}=n(n-1)+n^{2} H^{2}-S=n(n-1)-c_{0} .
$$

But if $M_{0}$ is totally umbilical, then $\rho_{0}=n(n-1)\left(H_{0}^{2}+1\right) \neq(n-1)(n-2)$ while for $M_{0}=M_{n-1,1}^{r_{0}}\left(H_{0}\right)$, we have $\rho_{0}=(n-1)(n-2) / r_{0}^{2} \neq(n-1)(n-2)$ for $0<r_{0}<1$. Hence, $n-1-(1 / 2) c_{0} \neq 0$ and we can conclude that $H= \pm H_{0}$. Therefore, $S=S_{0}$. Now, we can make use of Alencar-do Carmo's Theorem mentioned above to finish the proof of theorem.

Acknowledgments. The author would like to thank Abdênago Barros, Aldir Brasil and Jorge Lira for careful readings and helpful sugestions during the preparation of this work.

\section{REFERENCES}

[1] H. Alencar and M. do Carmo, Hypersurfaces with constant mean curvature in spheres, Proc. of the Amer. Math. Soc. 120 (1994), 4, 1223-1229.

[2] S. Almeida AND F. Brito, Closed 3-dimensional hypersurfaces with constant mean curvature and constant scalar curvature, Duke Math. J. 61 (1990), 195-206.

[ 3 ] M. Berger, P. Gauduchon et E. Mazet, Le Spectre d'une Variété Riemannienne, Lecture Notes in Mathematics, 194, Springer-Verlag, 1971.

[4] E. CARTAN, Sur des familles remarquables d'hypersurfaces isoparamétriques dans les espaces sphériques, Math. Zeitschrift 45 (1939), 335-367.

[5] S. Chang, A closed hypersurface with constant scalar and mean curvature in $S^{4}$ is isoparametric, Communications in Analysis and Geometry 1 (1993), 71-100.

[6] S. Chern, M. do Carmo and S. Kobayashi, Minimal submanifolds of a sphere with second fundamental form of constant length, Functional Analysis and Related Fields, SpringerVerlag, New York (1970), 59-75.

[ 7] Q. Ding, On spectral characterization of minimal hypersurfaces in a sphere, Kodai Math. J. 17 (1994), 320-328.

[ 8 ] U. K. Patodi, Curvature and the fundamental solution of the heat operator, J. Indian Math. Soc. 34 (1970), 269-285.

[9] B. L. van der Waerden, Algebra, Vol. 1, Springer-Verlag, New York (1991). 
[10] J. WANG, On spectral characterizations of isoparametric hypersurfaces in $S^{4}$, J. Math. Exposition 17 (1997), 496-500.

[11] S.-Y. Cheng And S.-T. YaU, Hypersurfaces with constant scalar curvature, Math. Ann. 225 (1977), 195-204.

Departamento de Matemática

Universidade Federal da BaHia

40.170-110 SALVAdOR-BA, Brazil

E-mail: jnelson@ufba.br 